Феоктистов С. И., Чжо Заяр Со

S. I. Feoktistov, Kyaw Zayar Soe

ОПРЕДЕЛЕНИЕ ТЕХНОЛОГИЧЕСКИХ ВОЗМОЖНОСТЕЙ ТИТАНОВЫХ И АЛЮМИНИЕВЫХ СПЛАВОВ ПРИ РАЗДАЧЕ

\title{
DETERMINATION OF TECHNOLOGICAL POSSIBILITIES OF TITANIUM AND ALUMINUM ALLOYS AT DISTRIBUTION
}

Феоктистов Сергей Иванович - доктор технических наук, профессор, декан самолётостроительного факультета Комсомольского-на-Амуре государственного университета (Россия, Комсомольск-на-Амуре); 681013, Хабаровский край, г. Комсомольск-на-Амуре, пр. Ленина, 27. E-mail: ssf@knastu.ru.

Mr. Sergey I. Feoktistov - Doctor of Engineering, Professor, Dean of the faculty of aircraft of Komsomolsk-onAmur State University (Russia, Komsomolsk-on-Amur); 681013, Khabarovsk territory, Komsomolsk-on-Amur, 27 Lenin str. E-mail: ssf@knastu.ru.

Чжо Заяр Со - аспирант Комсомольского-на-Амуре государственного университета (Россия, Комсомольск-на-Амуре); 681013, Хабаровский край, г. Комсомольск-на-Амуре, пр. Ленина, 27. E-mail: kyawzayarsoe52@gmail.com.

Mr. Kyaw Zayar Soe - post-graduate student of Komsomolsk-on-Amur State University (Russia, Komsomolskon-Amur); 681013, Khabarovsk territory, Komsomolsk-on-Amur, 27 Lenin str. E-mail: kyawzayarsoe52@gmail.com.

Аннотация. Разработан метод определения предельного коэффициента раздачи для титановых и алюминиевых сплавов, позволяющий достаточно точно определять момент разрушения заготовки. Метод основан на использовании диаграмм предельного формоизменения (Forming Limit Diagram - FLD) при прогнозировании разрушения заготовки, а также на использовании метода переменных параметров упругости при определении напряжённо-деформированного состояния в процессе раздачи.

Summary. The paper describes a method, which has been developed, for obtaining the limiting expanding ratio of titanium and aluminum alloys and determines the moment of failure of the workpiece. This method is based not only on the use of Forming Limit Diagram (FLD) in predicting the failure of the blank, but also on the using method of variable parameters of elasticity in determining the stress-strain state in expanding process.

Ключевые слова: FLD-диаграмма, диаграмма предельного формоизменения, раздача, метод переменных параметров упругости, технологические возможности.

Key words: FLD-diagram, forming limit diagram, expanding, method of variable parameters of elasticity, technological capabilities.

УДК 621.73.01/07.002

\section{Введение}

Одной из важнейших задач при разработке технологического процесса листовой штамповки-раздачи является прогнозирование момента разрушения заготовки, то есть определение предельного коэффициента раздачи [2]. Современный уровень развития вычислительной техники, её доступность для инженерных расчётов при проектировании процессов листовой штамповки позволяют с высокой точностью рассчитывать параметры напряжённо-деформированного состояния заготовки. Это даёт возможность прогнозировать разрушение заготовки с помощью диаграмм предельного формоизменения (Forming Limit Diagram - FLD) $[5 ; 11 ; 14 ; 7 ; 6 ; 10 ; 8]$.

Существует достаточно много способов получения диаграмм предельного формоизменения. Все они сводятся к достижению разрушения листового материала при различных предсказуемых или измеримых деформированных состояниях. Наиболее распространены методы Наказимы (K. Nakazima) [13] и Марчиньяка (Z. Marciniak) [12]. 
В нашей работе предлагается простой аналитический способ построения FLD-диаграммы с использованием справочных механических характеристик металла, основанный на математической модели разрушения бездефектных листовых изотропных заготовок.

При определении напряжённо-деформированного состояния используется метод переменных параметров упругости, позволяющий учитывать не только изменение толщины в процессе деформирования, но и упрочнение материала, которое для титановых и алюминиевых сплавов хорошо описывается степенной функцией.

Модель разрушения бездефектных листовых изотропных заготовок на основе геометрического критерия

При построении модели разрушения бездефектных листовых изотропных заготовок в качестве критерия предельного деформирования используется геометрический критерий локализации деформации, предсказывающий локальное интенсивное утонение заготовки, математическая запись которого имеет вид $\frac{d S}{d \rho}= \pm \infty$, где $d S-$ изменение толщины стенки заготовки по её длине $H$ в направлении действия наибольшего растягивающего напряжения.

Рассматривается процесс формоизменения листовой заготовки под действием хотя бы одного растягивающего напряжения $\left(\sigma_{1}\right)$, действующего в плоскости листа при условии, что ширина заготовки по её длине $H$ постоянная $(d l / d H=0)$, а заготовка может иметь исходную разнотолщинность $S_{c}=\varphi\left(H_{c}\right)$.

Решение этой задачи представлено в работах А. С. Чумадина [4].

Для случая монотонного деформирования бездефектных (равнотолщинных) заготовок математическая модель предельного деформирования имеет вид

$$
D \frac{\sigma_{1}}{S}-\sigma_{1} C\left(2 \sigma_{2}-\sigma_{1}\right)+\frac{4 \sigma \frac{2}{S} n\left(2 \mathrm{e}_{3}+\mathrm{e}_{2}\right)}{3 S e_{i}^{2}}=0,
$$

где

$$
\begin{aligned}
& C=\frac{3 e_{2}}{\left(-2 e_{3}-e_{2}\right)^{2}} ; D=\left(2 \sigma_{1}-\sigma_{2}\right)+B\left(2 \sigma_{2}-\sigma_{1}\right) ; \\
& \sigma_{1}=+\sqrt{\frac{\sigma_{s}^{2}}{(B)^{2}-B+1}} ; \sigma_{2}= \pm \sqrt{\frac{\sigma_{s}^{2}(B)^{2}}{(B)^{2}-B+1}} ; B=\frac{\sigma_{2}}{\sigma_{1}} .
\end{aligned}
$$

В этих уравнениях главные логарифмические деформаций равны:

$$
e_{1}=\ln \frac{d H}{d H_{c}} ; e_{2}=\ln \frac{L}{L_{c}} ; e_{3}=\ln \frac{S}{S_{c}}
$$

где $S_{c}, L_{c}$ - соответственно начальная толщина и ширина заготовки на длине $H_{c}, S, L-$ то же на длине $H$ в момент начала разрушения (образования шейки).

Для преобразования уравнения (1) используем основные физические зависимости, связывающие главные напряжения и главные деформации:

1) уравнение, описывающее состояния материала (кривая деформационного упрочнения) [1] в виде $\sigma_{s}=A e_{i}^{n}$, где $A, n$ - константы материала; $e i-$ интенсивность логарифмических деформации, причём $e_{i}=\frac{2}{\sqrt{3}} \sqrt{e_{1}^{2}+e_{1} e_{2}+e_{2}^{2}}$; 


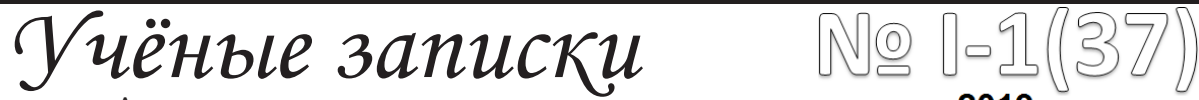 \\ Комсомольского-на-Амуре государственного технического университета 2019}

2) энергетическое условие пластичности: $\sigma_{s}^{2}=\sigma_{1}^{2}-\sigma_{1} \sigma_{2}+\sigma_{2}^{2}$, где $\sigma_{s}-$ напряжение сопротивления деформированию; $\sigma_{1} \sigma_{2}$ - главные напряжения;

3) условие постоянства объёма в главных логарифмических деформациях: $e_{1}+e_{2}+e_{3}=0$;

4) уравнение связи напряжений и деформаций: $\frac{\sigma_{2}}{\sigma_{1}}=\frac{2 e_{2}+e_{1}}{2 e_{1}+e_{2}}$.

Выражая входящие в уравнение (1) величины через $e_{1}$ и $e_{2}$, получим уравнение, описывающее кривую предельного формоизменения в координатах $e_{1}$ и $e_{2}$ :

$$
2\left(e_{1}^{2}+e_{1} \cdot e_{2}+e_{2}^{2}\right)\left(2 e_{1}+e_{2}\right)-3 e_{2}^{2}-n\left(2 e_{1}+e_{2}\right)^{2}=0 .
$$

В уравнении (2) $n$ - показатель степени, описывающий кривую деформационного упрочнения. Из [4; 3] известно, что если кривая упрочнения аппроксимируется степенной функцией, то для того чтобы эта аппроксимация удовлетворяла свойству кривых упрочнения 3-го рода, необходимо соблюдение условия $n=e_{\text {шा }}$, где $e_{\text {ш }}$ - истинная (логарифмическая) деформация, соответствующая моменту образования шейки (переход от распределённой деформации к сосредоточенной).

\section{Определение напряжённо-деформированного состояния при раздаче}

Представлена математическая модель процессов раздачи трубных заготовок с учётом изменения толщины. Предложенная методика позволяет исследовать процессы раздачи по криволинейной оправке с подпором, когда наблюдается значительное местное утолщение материала.

Широкое применение при изготовлении различных деталей и узлов летательных аппаратов нашли технологические процессы формоизменения трубчатых заготовок, такие как раздача, выворот внутрь и наружу. Эти процессы эффективны только с использованием термической интенсификации. Такие операции, как раздача, в случае использования подпора могут сопровождаться значительным местным утолщением материала.

При решении задачи напряжения и деформации осредняются по толщине и относятся к срединной поверхности, как в безмоментной теории оболочек.

Уравнения равновесия элемента трубчатой заготовки в проекциях на нормаль и касательную к срединной поверхности в соответствии с рис. 1 имеют вид

$$
\begin{gathered}
\frac{d}{d \rho}\left(\rho S \sigma_{\rho} \sin \psi\right)=-\left(q_{n}+q_{\tau} t g \psi\right) \rho ; \\
\frac{d}{d \rho}\left(\rho S \sigma_{\rho}\right)=\rho S \sigma_{\varphi}-\frac{q_{\tau}}{\cos \psi} \rho,
\end{gathered}
$$

где $S$ - средняя толщина рассматриваемого элемента; $\sigma_{\rho}$ - радиальное напряжение; $\psi$ - угол между касательной осью и $\rho$, отсчитываемый против часовой стрелки; $q_{n}-$ проекция усилия на нормаль, направленную к центру кривизны; $q_{\tau}$ - проекция усилия на касательную, направленную в сторону увеличения $\rho ; \sigma_{\varphi}$ - тангенциальное напряжение.

Для удобства можно угол $\psi$ заменить углом $\alpha$ (см. рис. 1$)$.

Если происходит раздача на конус, это будет угол конусности. Учитывая, что при раздаче $q_{n}=q, q_{\tau}=-\mu q, \psi=270^{\circ}+\alpha$, систему (3) можно привести к одному уравнению в напряжениях:

$$
\frac{d}{d \rho}\left(\rho S \sigma_{\rho}\right)=S \sigma_{\varphi} \frac{(1+\mu \cdot \operatorname{ctg} \alpha)}{\rho}-S \sigma_{\rho} \frac{\left(1+\mu \frac{d \alpha}{d \rho}\right)}{\rho} .
$$




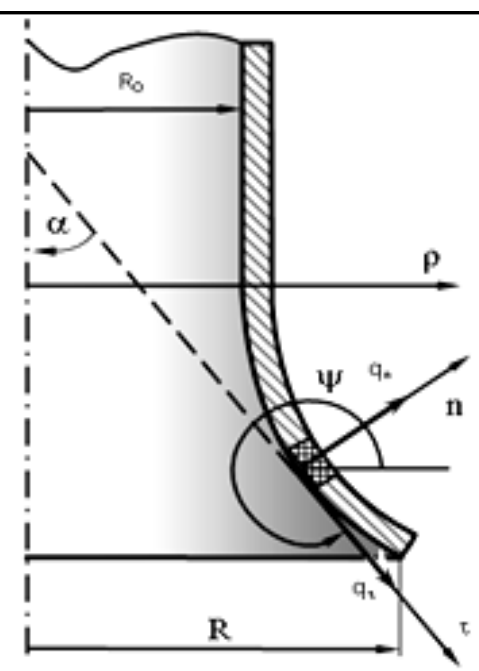

Рис. 1. Общий случай раздачи

Это уравнение описывает равновесие при раздаче.

Используя уравнение связи напряжений и деформаций в соответствии с гипотезами деформационной теории, получим интегральное уравнение равновесия в деформациях:

$$
\begin{gathered}
e_{\rho}=-\frac{1}{2} e_{\varphi}+\frac{3 U(\rho)}{4 S E_{\text {сек }}} \int_{R}^{\rho} \frac{S E_{\text {сек }} e_{\varphi}(1+\mu \cdot \operatorname{ctg} \alpha)}{\rho U(\rho)} d \rho+\frac{E_{\text {секR }} S_{R}}{E_{\text {сек }} S}\left(\mathrm{e}_{\rho R}+\frac{1}{2} e_{\varphi R}\right) U(\rho), \\
U(\rho)=\exp \left[-\int_{R}^{\rho} \frac{1-\mu \cdot \operatorname{ctg} \alpha+2 \mu \rho \frac{d \alpha}{d \rho}}{2 \rho} d \rho\right], \\
e_{\varphi}=\ln \frac{\rho}{R_{0}} .
\end{gathered}
$$

При рассмотрении раздачи в конической матрице угол $\alpha$ равен углу конусности $\alpha_{\kappa}$. Тогда уравнение (6) упрощается и принимает вид

$$
U(\rho)=\exp \left[\frac{1-\mu \cdot \operatorname{ctg} \alpha_{\mathrm{\kappa}}}{2} \ln \frac{R}{\rho}\right] .
$$

\section{Определение технологических возможностей при раздаче}

Кривую деформационного упрочнения титанового или алюминиевого сплава аппроксимируют степенной функцией в виде уравнения Холломона (J. H. Hollomon) [9]: $\sigma_{s}=\sigma_{s ш} \cdot\left(e_{i} / e_{\mathrm{m}}\right)^{e_{\mathrm{m}}}$, где $\sigma_{s ш}=\sigma_{B} \cdot \exp \left(e_{\mathrm{m}}\right)$, а $e_{\mathrm{m}}-$ истинная (логарифмическая) деформация, соответствующая моменту образования шейки (переход от распределённой деформации к сосредоточенной) и $\sigma_{B}-$ предел прочности материала (временное сопротивление при испытании на разрыв).

Технологический процесс раздачи задают следующими параметрами: $R_{0}$ - радиус заготовки; $\alpha_{\text {к }}$-угол конусности; $\mu$ - коэффициент трения и радиус наружного края $R$.

Наиболее полное представление о прохождении процесса раздачи даёт положение наружного края $R$, поэтому задают ещё шаг изменения положения наружного края $\Delta R$. 


\section{Учёнье записки \\ Комсомольского-на-Амуре государственного технического университета \\ NOC.9. ((5)) \\ 2019}

При заданном положении наружного края при использовании метода переменных параметров упругости [1] определяют напряжённо-деформированное состояние фланца заготовки в следующем порядке:

1. В первом приближении принимают толщину заготовки постоянной и равной начальной толщине, а секущий модуль - равным модулю упругости; определив $U(\rho)$ и $e_{\varphi}$ по формулам (6) и (7), подставляют полученные значения в (5) и численно решают интегральное уравнение для нахождения $e_{\varphi}$.

2. После определения деформированного состояния находят напряжённое состояние и уточняют значения толщины и секущего модуля, затем расчёт повторяют снова до тех пор, пока значение секущего модуля последующего приближения не будет достаточно близко к предыдущему. Как показали расчёты, метод обладает хорошей сходимостью процесса последовательных приближений.

3. Затем, принимая $e_{\mathrm{\rho}}=e_{1}$, а $e_{\varphi}=e_{2}$, определяют положение точек, характеризующих деформированное состояние фланца в координатах $e_{1}, e_{2}$. Если все эти точки лежат ниже кривой предельного формоизменения, определяемой уравнением (2), то процесс продолжают, изменяя положение наружного края $R$. В случае если на каком-либо этапе нагружения одна из точек, характеризующих деформированное состояние фланца в координатах $e_{1}, e_{2}$, будет находиться выше диаграммы предельного формоизменения, то расчёт прекращают и определяют предельный коэффициент раздачи $K_{\text {раз }}=\frac{R_{0}}{R}$.

Результаты расчётов для материала ВТ1-1 представлены на рис. 1, где кривые 1, 2 характеризуют деформированное состояние заготовки в момент, предшествовавший разрушению. Сплошная толстая линия соответствует кривой предельного формоизменения (FLC), построенной по уравнению (2).

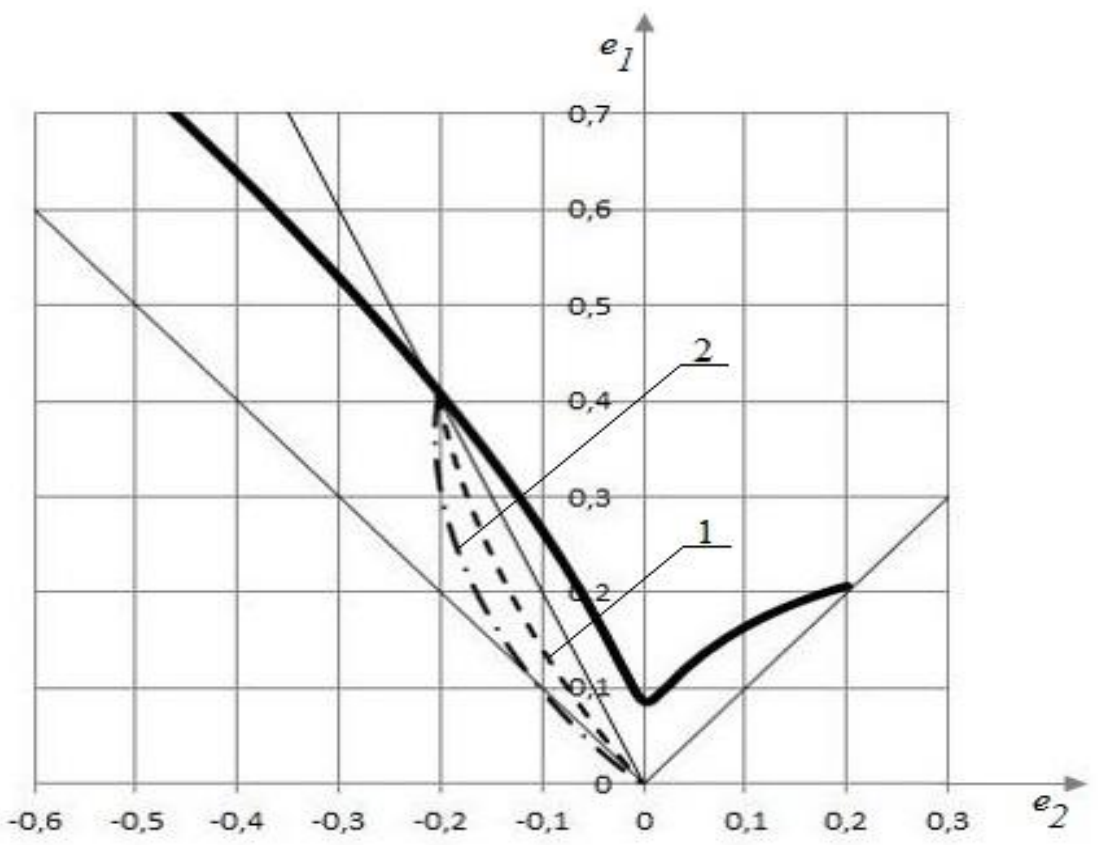

Рис. 1. Определение коэффициента раздачи для материала ВТ1-1 $\left(e_{\mathrm{m}}=0,086\right)$ :

1 - без учёта трения $\left(K_{\text {раз }}=0,67\right) ; 2-$ с учётом трения $(\mu=0,3),\left(K_{\text {раз }}=0,67\right)$ 


\section{Вывод}

В результате исследования получены методы определения технологических возможностей титановых и алюминиевых сплавов при раздаче, позволяющие прогнозировать разрушение заготовки по диаграмме предельного формоизменения (Forming Limit Diagram - FLD).

\section{ЛИТЕРАТУРА}

1. Малинин, Н. Н. Прикладная теория пластичности и ползучести: учебник для вузов / Н. Н. Малинин. 2-е изд., перераб. и доп. - М.: Машиностроение,1975. - 400 с.

2. Романовский, В. П. Справочник по холодной штамповке / В. П. Романовский. - 5-е изд., перераб. и доп. М.: Машиностроение, 1977. -423 с.

3. Сторожев, М. В. Теория обработки металлов давлением: учебник для вузов / М. В. Сторожев, Е. А. Попов. - 4-е изд., перераб. и доп. - М.: Машиностроение, 1977. -423 с.

4. Чумадин, А. С. Теория и расчёты процессов листовой штамповки (для инженеров) / А. С. Чумадин. М: Экспосервис «ВИП», 2014. - 216 с.

5. Banabic, D. Sheet Metal Forming Processes. Constitutive Modelling and Numerical Simulation / D. Banabic. London - New York: Springer Heidelberg Dordrecht, 2010. - 301 p.

6. Chen, G. N. Damage, Instability and Construction of Forming Limit Curve in Sheet Metal Stretch Forming G. N. Chen, S. G. Hu // J. Journal of Plasticity Engineering. - 1994. - Vol. 1. - P. 31-46.

7. Du, P. M. Theoretical prediction and parameter influence of FLDs based on M-K model / P. M. Du, L. H. Lang, B. S. Liu // J. Journal of Plasticity Engineering. - 2011. - Vol. 18. - P. 84-89.

8. Ghosh, A. K. The influence of strain hardening and strain-rate sensitivity on sheet metal forming [J] A. K. Ghosh // Journal of Engineering Materials and Technology. - 1977. - Vol. 99(3). - P. 264-274.

9. Hollomon, J. H. Tensile deformation, transaction of American Institute of Mechanical Engineering / J. H. Hollomon. - 1945. - Vol. 162. - P. 268-277.

10. Kami, A. Numerical determination of the forming limit curves of anisotropic sheet metals using GTN damage model / A. Kami, B. M. Dariani // J. Journal of Materials Processing Technology. - 2015. - Vol. 216. - P. 472-483.

11. Ma, G. S. Theoretical prediction of FLDs for Al-Li alloy at elevated temperature based on M-K model / G. S. Ma, M. Wan, X. D. Wu // Chinese Journal of Nonferrous Metals. - 2008. - Vol. 18. - P. 980-984.

12. Marciniak, Z. Limit strain in the process of stretch forming sheet metal / Z. Marciniak, K. Kucznski // International Journal of Mechanical science. - 1967. - Vol. 9. - P. 609-620.

13. Nakazima, K. Forming Limits under biaxial stretching of sheet metals / K. Nakazima, T. Kikuma // Testu-to Hagane. - 1967. - Vol. 53. - P. 455-458.

14. Yang, M. The Forming Limit Prediction Using a Rate-Dependent Crystal Plasticity Model X.cH. Dong M. Yang // J. Journal of ShangHai JiaoTong University. - 2008. - Vol. 42. - P. 720-723. 